\title{
Development of synchrotron pink beam x-ray grating interferometer at the Diamond Light source I13-2 beamline
}

Shashidhara Marathe, Malte Storm, Venkata S. C. Kuppilli, Richard Harrison, Gunjan Das, et al.

Shashidhara Marathe, Malte Storm, Venkata S. C. Kuppilli, Richard Harrison, Gunjan Das, Sven L. M. Schroeder, Silvia Cipiccia, Florian Döring, Christian David, Christoph Rau, "Development of synchrotron pink beam x-ray grating interferometer at the Diamond Light source I13-2 beamline," Proc. SPIE 11113, Developments in X-Ray Tomography XII, 1111319 (16 October 2019); doi: $10.1117 / 12.2530698$

Event: SPIE Optical Engineering + Applications, 2019, San Diego, California, United States 


\title{
Development of a Synchrotron Pink Beam X-ray Grating Interferometer at the Diamond Light Source I13-2 Beamline
}

\author{
Shashidhara Marathe*a, Malte Storm ${ }^{\mathrm{a}}$, Venkata S. C. Kuppili ${ }^{\mathrm{b}}$, Richard Harrison ${ }^{\mathrm{b}}$, Gunjan Das $^{\mathrm{a}, \mathrm{c}, \mathrm{d}}$, \\ Sven L. M. Schroeder ${ }^{\mathrm{a}, \mathrm{c}, \mathrm{d}}$, Silvia Cipiccia ${ }^{\mathrm{a}}$, Florian Döring ${ }^{\mathrm{e}}$, Christian David ${ }^{\mathrm{e}}$ and Christoph Rau ${ }^{\mathrm{a}}$ \\ aDiamond Light Source, Harwell Science and Innovation Campus, Didcot, OX11 0DE, UK, \\ ${ }^{\mathrm{b}}$ University of Cambridge, Cambridge, UK, ${ }^{\mathrm{c} S}$ School of Chemical and Process Engineering, \\ University of Leeds, Leeds, UK, ${ }^{\mathrm{d}}$ Research complex at Harwell, Rutherford Appleton Laboratory, \\ Didcot, OX11 0FA, 'Paul-Scherrer-Institut (PSI), Laboratory for Micro and Nanotechnology, 5232 \\ Villigen, Switzerland
}

\begin{abstract}
X-ray Grating Talbot Interferometer (XGTI) is already routinely used for quantitative phase contrast imaging of soft tissue samples. XGTI can be realized using various measurement techniques, depending on the X-ray source used, the required spatial resolution and the speed of acquisition. The phase-stepping measurement technique, which is commonly used for XGTI data acquisition, needs multiple acquisitions for a single projection. For fast imaging the Moiré technique, a single-shot technique, is often preferred. However, it requires two gratings which increases the dose on the sample. We have therefore examined the Spatial Harmonic Imaging (SHI) technique which is low-dose and single-shot, using the I13-2 Diamond-Manchester Beamline at Diamond Light Source (DLS). The DLS I13-2 beamline is equipped with a Double Crystal Monochromator (DCM) and a Multi-Layer Monochromator (MLM) to deliver monochromatic beam, which work at the energy bandwidths $(\Delta E / E)$ of $10^{-4}$ and $10^{-2}$, respectively. However, the disadvantage of using these monochromators, especially for fast imaging, is loss of X-ray flux. It has already been shown that XGTI can work with an energy bandwidth $(\Delta E / E)$ of $10^{-1}$. Our aim is to develop a single phase grating interferometer with pink beam from an undulator source, with X-ray mirror optics and multiple absorption filters, to obtain maximum possible flux with sufficient coherence and monochromaticity. We demonstrate performance with optimized beamline parameters for a photon energy of $15 \mathrm{keV}$ with some demonstrative image reconstructions.
\end{abstract}

Keywords: X-ray grating interferometer, Phase-contrast imaging, Spatial Harmonic Imaging, Tomography, Fast imaging, Pink-beam

\section{INTRODUCTION}

XGTI is a phase-contrast imaging technique that can provide three image contrast modes from a single interferogram. Besides standard absorption contrast, it provides Differential Phase Contrast (DPC). Combination of these two contrast modes is very useful when the sample has multiple phases, for example highly absorbing components combined with slightly absorbing or purely phase-sensitive materials. ${ }^{1-5}$ The third contrast mode that we can obtain from XGTI is the Small Angle X-ray Scattering (SAXS) contrast which manifests itself as reduced visibility of the interference pattern due to presence of the sample. It gives access to spatial information below the pixel size of the detector. Unlike other phase contrast imaging techniques, XGTI is a quantitative phase-imaging technique ${ }^{1}$ and the requirements on the temporal resolution are moderate. It has been shown that grating interferometer can be realized with an energy spread of about \pm 2 $\mathrm{keV}^{5}$

XGTI has been used with variety of X-ray sources. An X-ray tube source was used in combination with three transmission gratings to demonstrate XGTI for the first time. ${ }^{6}$ Later it was performed with a liquid-metal-jet source ${ }^{7}$ and recently with micro computed tomography system phoenix nanotom ${ }^{\circledR} \mathrm{m}^{8}$ (Universal Systems, US). At synchrotron sources, using a monochromatic X-ray source, such as MLM or DCM for fast imaging has the limitation of lower flux even though it gives the highest contrast images. Using synchrotron white beam from a wiggler source, Momose et al. have shown that high-speed X-ray phase imaging is possible. ${ }^{4}$ The other aspect of building an XGTI for fast imaging is the measurement technique. A proof of principle experiment was first reported by Takeda et al. ${ }^{9}$, demonstrating phasestepping XGTI with a single phase grating. Although this reduces the total acquisition time, multiple images per projection are still required. A recent report, quantitatively comparing this type of single grating XGTI with the double

*Shashidhara.marathe@diamond.ac.uk; phone 44 (0)1235 778663

Developments in X-Ray Tomography XII, edited by Bert Müller, Ge Wang, Proc. of SPIE

Vol. 11113, $1111319 \cdot$ - () The Authors. Published under a Creative Commons

Attribution CC-BY 3.0 License · doi: 10.1117/12.2530698 
grating, demonstrates that even though the contrast-to-noise ratio is lower for single grating XGTI, the spatial resolution is twofold better. ${ }^{10}$ To observe processes occurring at a few tens of millisecond time-frames it is desirable to have an even quicker data measurement technique. Therefore, we have explored the idea of using a single harmonic peak from a synchrotron undulator X-ray source by filtering the pink beam, for fast or dynamic imaging. In conjunction with the filtered X-ray pink beam, we also used the single grating SHI technique ${ }^{3,11,12}$ to speed up the data acquisition. The XGTI system described below can reconstruct data using only a single interferogram, and can therefore be used for dynamic imaging. However, the drawback is that spatial resolution is limited since the harmonic images extracted from the Fourier transform of the interferogram that are band limited.

\section{EXPERIMENTAL SETUP}

In this section, we describe the fundamental principles of grating interferometer, its operating conditions, and additional settings we used for generating the filtered pink X-ray beam, including the data processing technique.

\subsection{X-ray grating Talbot interferometer}

XGTI works on the principle of the Talbot effect ${ }^{13}$ which is a near-field diffraction phenomenon. A plane wave incident on a periodic structure, such as phase or absorption grating, propagates downstream such that it replicates the structure at a periodic distance from it. These periodic positions where there is revival of the grating structure are called Talbot distances or fractional Talbot distances $\left(d_{f t}\right)$. A phase grating with $\pi$ or $\pi / 2$ phase-shifting structures are usually preferred since they create a rectangular pattern with a maximum visibility. For a $1 \mathrm{D} \pi / 2$ phase-shifting grating, $d_{f t}$ is given by

$$
d_{f t}=\frac{n p^{2}}{2 \eta^{2} \lambda},
$$

for $n=1,3,5 \ldots, \eta=1$, where $p$ is the period of the grating, and $\lambda$ the X-ray wavelength. Typically, the interference pattern period is of a few microns in size. A detector placed at this maximum visibility position can be used to collect the interferograms. Since we do not have a second grating, we need to use a high spatial resolution detector system to resolve the grating structure of the interferogram. Processing the interferograms, measured with and without sample in the beam, produces three different contrast modes of the grating interferometer. There are different types of measurement and data analysis techniques that are available, but our application was for fast/dynamic imaging, hence we used the SHI technique. A brief description of the latter is provided in Section 2.3.

\subsection{Filtered X-ray beam (Pink Beam)}

The I13 beamline at DLS is located on one of the long straight sections of the storage ring with a mini-beta layout giving space for two branchlines: I13-2 Imaging and I13-1 Coherence ${ }^{14}$. Since we are using an undulator source, there are periodic peaks in the flux versus energy curve. These peaks can be shifted by changing the magnetic field by altering the Insertion Device (ID) gap. Therefore, by changing the ID gap we can get one of the harmonics to peak at a particular energy. In addition, we can use the X-ray reflecting mirror to attenuate part of the spectrum. Fig. 1 in reference 15 shows an example of this setup for $15 \mathrm{keV}$. Here the X-ray beam from the undulator source passes through the attenuating filters and is then reflected by a silicon mirror to obtain a strong peak at $15 \mathrm{keV}$. We have carbon filters to absorb very low X-ray energies and aluminum filters which attenuate X-rays up to approximately $15 \mathrm{keV}$. In addition, we have yittrium filter which has $\mathrm{K}$-edge at $17 \mathrm{keV}$ to suppress all energies lower than $15 \mathrm{keV}$. At an ID gap of approximately $11.09 \mathrm{~mm}$, one of the harmonics has a peak at around $15 \mathrm{keV}$ and all the other harmonics are suppressed due to the combination of filters and the Si reflective mirror. The dashed blue line shows the effective filter transmission, whereas the dashed violet line shows the mirror reflectivity. The solid red curve represents the effective spectrum arising from the combination of filters, mirror reflectivity and ID gap setting. The undulator spectrum and the X-ray spectrum with filters were generated using the Spectra software ${ }^{16}$, a standalone application for calculating various characteristics of synchrotron radiation.

\subsection{The Spatial Harmonic Imaging (SHI) technique}

Principles of the SHI technique were detailed elsewhere, ${ }^{3,11,12}$ so we restrict ourselves to presenting a brief account of the technique. In SHI technique, a high resolution detector system is used at a fractional Talbot distance to measure the interferograms directly to resolve the grating structure. A sample placed in the beam attenuates and refracts the interferogram, which is proportional to the product of the transmittance of the object with the grating pattern ${ }^{11}$. Fourier transform of a given projection image (which gives a convolution of the sample spectrum with that of the grating grid 
structure), we have one primary peak centered at the spatial frequency origin and at least two harmonic peaks adjacent to the primary peak. An inverse Fourier transform of a band-pass-limited image of the primary peak produces an image which is sensitive to only transmitted amplitude. This can be used to calculate the attenuation image. A band-passlimited image of the $1^{\text {st }}$ harmonic peak is a complex conjugate image, which implies that they are sensitive to both amplitude and phase signal. Since amplitude of the $1^{\text {st }}$ harmonic represents the reduction of the total amplitude from scattering, comparing the amplitude of the primary and $1^{\text {st }}$ harmonics gives the dark-field (DK) contrast image. The argument of this complex conjugate image provides the DPC image. One of the most valuable aspects of the SHI technique for fast imaging is that we can get all three contrast modalities from a single interferogram, albeit at the cost of reduced spatial resolution. ${ }^{3}$

\section{EXPERIMENT}

Our experiment was performed at the I13-2 Imaging Branchline at DLS, using filtered pink X-ray beam, with energy centered around $15 \mathrm{keV}$ and a spread of $\sigma \sim 2 \mathrm{keV}$ produced from a combination of a Si mirror and X-ray filters. We used the following X-ray filters: carbon $(1.3 \mathrm{~mm})$, aluminum $(1.8 \mathrm{~mm})$ and yittrium $(50 \mu \mathrm{m})$. The ID gap was set to $11.09 \mathrm{~mm}$ to produce an undulator harmonic peak at that energy as described in section 2.1. A 1D phase grating of $4.785 \mu \mathrm{m}$ nominal period was used, which gives a $3 \pi / 2$ phase shift at $15 \mathrm{keV}$. The detector was placed at $14 \mathrm{~cm}$ from the phase grating, corresponding to the $1^{\text {st }}$ fractional Talbot distance. Pco.edge 5.5 (PCO AG, Germany) camera was used with a $4 \mathrm{x}$ objective lens. Taking into account the magnification created by the objective and the optical components of the detector system (giving 8x total magnification), the effective size of a single pixel of the detector was $0.81 \times 0.81 \mu \mathrm{m}$. The visibility of the interferograms measured with a $4 x$ magnification objective was $14.6 \pm 0.5 \%$.

\section{RESULTS AND DISCUSSION}

The main objective of the study was to demonstrate the use of filtered pink beam to perform grating interferometer experiment, and most importantly for fast imaging. A filtered pink beam provides about 10-20 times more flux than the normal double crystal monochromator beam. At I13-2, the pink beam mode is stable which is essential for dynamic experiments as any beam movement or instability is not easy to distinguish from sample movement when a sample fills the whole field of view of the detector system. At some X-ray energies, the filtered pink beam flux is comparable to that of the MLM. Results from a static sample measured in this beam mode are shown in the following section. In section 4.2 below, we elucidate the application of this system to fast imaging, in this case to the anti-solvent crystallization of the amino acid glycine.

\subsection{Filtered pink beam static XGTI experiment}
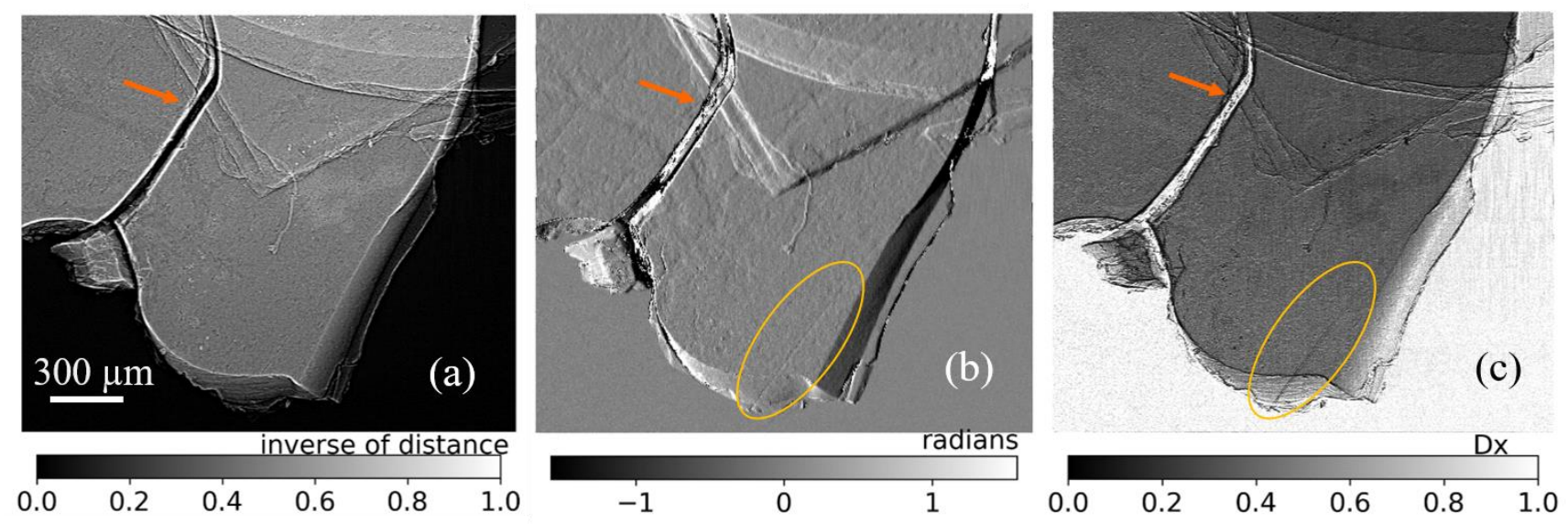

Figure 1. Three different contrast modalities of grating interferometer images of a silicified Precambrian limestone rock sample. (a) Linear attenuation coefficient, (b) DPC, (c) DK signal providing additional information. The images also show a stable background signal. 
Initially, we conducted an experiment with a static sample to evaluate the performance of the system. A silicified Precambrian limestone sample from NW Canada was chosen for this evaluation. Fig. 1 shows the reconstructed images from this sample. Fig 1(a) shows the linear attenuation coefficient calculated from the transmission image showing a flat background signal. The X-ray mirror that deflects the beam is horizontally reflecting, so any stability issue with the mirror would manifest as a smearing of the interference pattern. We did not perform any exclusive measurement of the stability, but over the course of measuring 2000 images we observed the interference pattern was stable. Fig. 1(b) and 1(c) show the DPC and DK images respectively. These images show a crack, as indicated by an orange oval shape on the DPC and DK images, which is not clearly evident in the attenuation image. Clearly, the DPC and DK images contain more information including - but not limited to - the crack indicated by the orange oval; the various features pointed out by the red arrow mark are not visible in Fig 1(a).

The DPC image has high contrast, suggesting that beam transverse coherence is sufficient to reconstruct the images. The gray scale bar unit Dx in Fig 1c is the usual measure for describing the dark-field signal, and it represents the normalized visibility of the sample image with respect to background image. As indicated by the gray scale values outside of the sample area in the DK image, the normalized amplitude is maximum, indicating very low scattering in this area and minimum around the edges where the most scattering occurs. Note that the images in Fig. 1 were obtained without any filters being applied for smoothing. In the future, we plan to carry out a more thorough test to compare these results with this sample in a monochromatic beam condition.

\subsection{Filtered pink beam dynamic XGTI experiment}

Crystallization is an important process for manufacturing across the whole chemical and pharmaceutical industry sector. Traditional industrial crystallizers are fixed batch vessels, which present intrinsic limits to scale-up (and scale-down) processes and the implementation of product and process variations. Continuous crystallization is seen as a possible route forward to overcome these challenges.

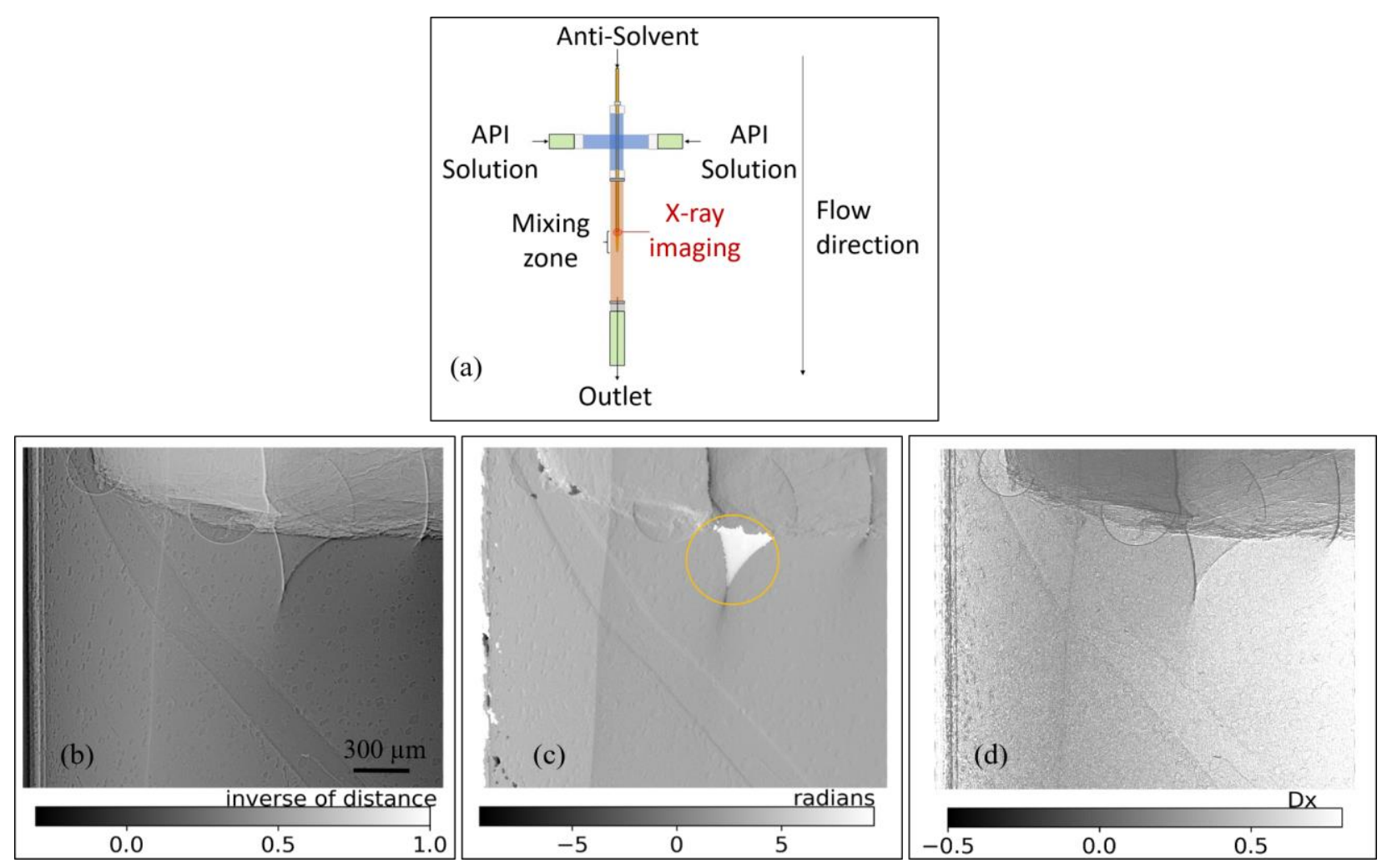

Figure 2. (a) Schematic of the flow crystallizer. (b,c,d) Reconstructed images from a single interferogram from XGTI dynamic measurement of the crystallization process. (b) The attenuation image shows the two fluids mixing at the entrance of the inner tube. (c) The DPC image shows wrapping at the mixing cone, indicating higher contrast of this region. (d) The DK contrast shows all the interfaces much clearly. 
The aim of this work was to improve the understanding of the physical processes taking place during the early stages of a continuous crystallization process producing organic crystals from a liquid solution. The dynamic nature of this process requires fast imaging. We have carried out an XGTI experiment using a concentric mixing flow crystallizer with an outer Kapton tube for X-ray transparency. The studies were carried out on an anti-solvent crystallization of glycine. Figure 2(a) shows a schematic of the setup. Flows of glycine solution, an amino acid ( $233 \mathrm{~g}$ glycine in $1 \mathrm{~kg}$ of water) and methanol (the anti-solvent) were combined. The solution was pumped through the outer tube and anti-solvent was pumped through the concentric inner tube.

As described in the experiment section, the grating was placed in-between the sample and the detector at a fractional Talbot distance. We acquired total of 178 images with $0.5 \mathrm{~s}$ exposure time per frame. Figs 2(b-d) show the reconstructed images from a single acquisition. The attenuation image shows the formation of a mixing zone (green circle) where the fluid from the inner tube mixes with fluid from the outer tube. In the attenuation image (Fig 2(b)), the contrast of the bubble formed right next to this mixing zone is very similar to that of the mixing zone, while the DPC image has very high contrast as indicated by a wrapped mixing zone. The DPC image after reconstruction was unwrapped however, the mixing zone area was not fully unwrapped. Nevertheless, the most important aspect of these results is that they provide spatiotemporally-resolved insight into the mixing process. The DK image, Fig 2(d), shows the different interfaces very clearly.
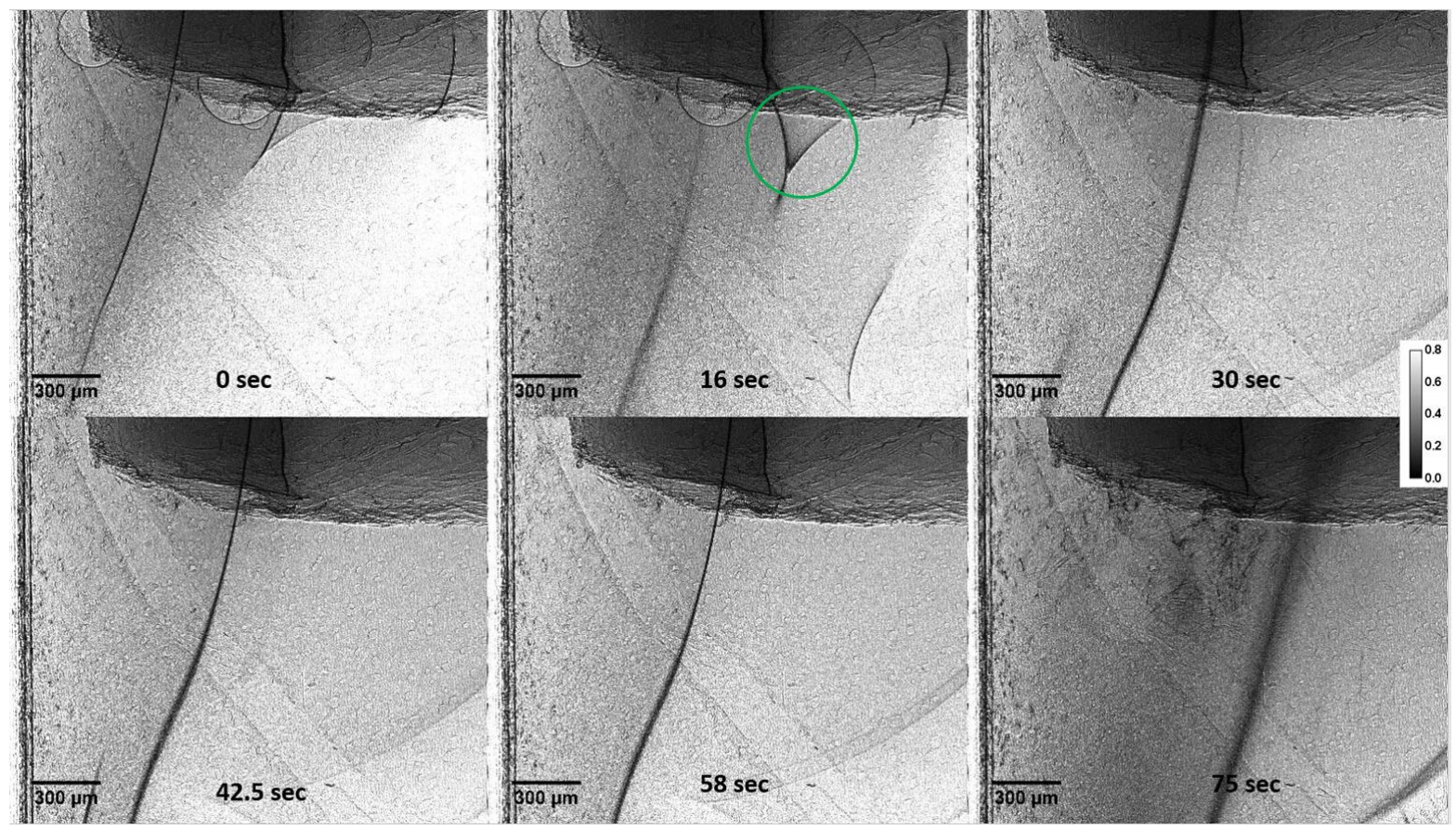

Figure 3. Time-lapse montage of images of the anti-solvent crystallization of glycine. The crystallization began with the formation of a mixing zone (at $16 \mathrm{~s}$ ), followed by phase separation (at $30 \mathrm{~s}$ ) and nucleation (58 s). Finally, by $75 \mathrm{~s}$ we saw crystal growth, which happened at the mixing zone.

Fig. 3 shows how XGI demonstrated for the first time a spatially resolved real-time visualization of the processes taking place in the mixing zone of a concentric-flow anti-solvent crystallizer. At $0 \mathrm{~s}$, we observed the flow of anti-solvent from the inner tubing and solution from the outer tubing forming an interface. At $16 \mathrm{~s}$ we clearly saw the mixing zone of solution, where we predict the crystallization takes place. At $30 \mathrm{~s}$ we were able to see phase separation in solute/solvent systems. By $58 \mathrm{~s}$ we were able to visualize crystal nucleation and by $75 \mathrm{~s}$, crystal growth. We were able to detect these unknown processes using the concentric device. This has opened up an exciting route to studying various active pharmaceutical ingredients (API) and their behavior during mixing and crystallization. Future work includes modifying the setup as well as taking images faster, to be able to visualize detailed information about crystal nucleation and growth. 
These experiments were focused on fast 2D imaging; the natural extension is to apply to tomography. We believe that it should in principle be possible to build a 3D image from a tomographic data set as with other types of grating interferometer.

\section{CONCLUSIONS}

We have demonstrated the operation of a filtered pink beam single X-ray Grating Talbot Interferometer, using a Precambrian limestone rock sample and anti-solvent crystallization. From the results it is evident that different contrast modes provide complementary information about the object. The filtered pink beam is stable with good intensity for dynamic experiments. For imaging nucleation and the initial stages of crystal growth, DK contrast is best suited. Future work will include testing different filtered pink beam energies, improving the intensity of the beam and demonstrating tomographic reconstruction.

\section{ACKNOWLEDGEMENTS}

The authors gratefully acknowledge the use of Diamond Light Source on the I13-2 Diamond-Manchester Branchline, during the experiment (MT21327) and the internal commissioning beamtime. We acknowledge Prof. N.J. Butterfield for providing the rock sample. SLMS acknowledges financial support by the Royal Academy of Engineering and Infineum UK through the Bragg Centenary Chair. GD and SLMS acknowledge the use of laboratory facilities available at the Research Complex at Harwell and with financial support from the Future Continuous Manufacturing and Advanced Crystallization (CMAC) Hub (EPSRC Grant EP/P006965/1). GD acknowledges the University of Leeds for the PhD studentship. We would also like to thank Dr. Kaz Wanelik, Ben Bradnick and Simon Logan, Diamond Light Source, UK, for their help during the experiments and data analyses.

\section{REFERENCES}

[1] Zdora, M-C., Vila-Comamala, J., Schulz, G., Khimchenko, A., Hipp, A., Cook, A. C., Dilg, D., David, C., Grünzweig, C., Rau, C., Thibault, P., Zanette, I., "X-ray phase microtomography with a single grating for highthroughput investigations of biological Tissue," Biomed. Opt. Express, 8, 1257-1270 (2017).

[2] Bikis, C., Rodgers, G., Deyhle H., Thalmann, P., Hipp A., Beckmann, F., Weitkamp, T., Theocharis, S., Rau C., Schulz, G., Müller, B., "Sensitivity comparison of absorption and grating-based phase tomography of paraffinembedded human brain tissue," Appl. Phys. Lett. 114, 083702 (2019).

[3] Marathe,S., Zdora, M-C., Zanette, I., Cipiccia, S., Rau, C., "Comparison of data processing techniques for singlegrating X-ray Talbot interferometer data," Proc. SPIE 10391, Developments in X-Ray Tomography XI, 103910S (2017).

[4] Momose, A., Yashiro W., Harasse S., Kuwabara H., "Four-dimensional X-ray phase tomography with Talbot interferometry and white synchrotron radiation: dynamic observation of a living worm," Opt. Express 19, 84238432 (2011).

[5] Weitkamp, T., Diaz, A., David, C., Pfeiffer, F., Stampanoni, M., Cloetens, P., Ziegler, E., "X-ray phase imaging with a grating interferometer," Opt. Express 13, 6296-6304 (2005).

[6] Pfeiffer, F., Weitkamp, T., Bunk, O., David, C., "Phase retrieval and differential phase-contrast imaging with lowbrilliance X-ray sources," Nat. Phys., 2, 258-261 (2006).

[7] Thuring, T., Zhou, T., Lundstrom, U., Burvall, A., Rutishauser, S., David, C., Hertz., H. M., Stampanoni, M., "Xray grating interferometry with a liquid-metal-jet source," Appl. Phys. Lett. 103, 091105 (2013).

[8] Khimchenko, A., Schulz, G., Thalmann, P., Müller, B., "Implementation of a double-grating interferometer for phase-contrast computed tomography in a conventional system nanotom® m," APL Bioeng. 2, 016106 (2018).

[9] Takeda, Y., Yashiro, W., Suzuki, Y., Aoki, S., Hattori, T., Momose, A.,"X-ray phase imaging with single phase grating," Jpn. J. Appl. Phys. 46, L89 (2007).

[10] Thalmann, P., Bikis, C., Hipp, A., Müller, B., Hieber, S. E., Schulz, G., "Single and double grating-based X-ray microtomography using synchrotron radiation", Appl. Phys. Lett. 110, 061103 (2017).

[11] Wen, H. H., Bennett, E. E., Kopace, R., Stein, A. F., Pai, V., "Single-shot x-ray differential phase-contrastand diffraction imaging using two-dimensional transmission gratings," Opt. Lett., 35, 1932-1934 (2010).

[12] Marathe, S., Shi, X., Wojcik, M. J., Kujala, N. G., Wen, H., Divan, R., Mancini, D. C., Macrander, A. T., and Assoufid, L., "Probing transverse coherence of X-ray beam with 2-D phase grating interferometer," Opt. Express 22, 14041 (2014). 
[13] Talbot, H. F., "LXXVI. Facts relating to optical science. No. IV,” Philos. Mag. 9, 401-407 (1836).

[14] Rau, C., Wagner, U., Pesic, Z., Fanis, A. D., "Coherent imaging at the Diamond beamline I13," Phy. Status Solidi A -Applications and Materials Science, 208(11), 2522-2525 (2011).

[15] Rau, C., Storm, M., Marathe, S., Bodey, A. J., Zdora, M., Cipiccia, S., Batey, D., Shi, X., Schroeder, S. M. L., Das, G., Loveridge, M., Ziesche, R., Connolly, B., Proc. SPIE 11113, Developments in X-Ray Tomography XII, $111130 \mathrm{P}(2019)$.

[16] Tanaka, T., Kitamura, H., "SPECTRA: a synchrotron radiation calculation code," J. Synchrotron Rad. 8, 12211228 (2001).

[17] Svoboda, V., MacFhionnghaile, P., McGinty, J., Connor, L. E., Oswald I. D. H., Sefcik, J., "Continuous Cocrystallization of Benzoic Acid and Isonicotinamide by Mixing-Induced Supersaturation: Exploring Opportunities between Reactive and Antisolvent Crystallization Concepts," Cryst. Growth \& Des., 17, 1902-1909 (2017). 